\title{
MRI for assessment of pathologic nipple discharge: is it mandatory?
}

Mona M. Zaky ${ }^{1}$, Asmaa Hafez ${ }^{1}$, Marwa M. Zaky², Ashraf Shoma ${ }^{3}$, Nermin Y. Soliman and Ali H. Elmokadem(i)

\begin{abstract}
Background: Benign breast lesions is the most common cause of nipple discharge; however, a rare but major cause is breast cancer. This study assesses the superadded value of MRI in diagnosing causes of pathologic nipple discharge. Ninety-three patients with pathologic nipple discharge were evaluated by sonomammography and DCE-MRI. Sonomammography and MR imaging features were analyzed and correlated with the histopathology.

Results: Histopathology revealed 69 benign, three high-risk, and 21 malignant lesions. Simply dilated ducts and presence of a mass on US examination as well as non-mass enhancement and STIR signal changes on MRI were of statistically significant probability in differentiation between benign and malignant causes of pathological nipple discharge $(p$ value $=0.017$ and 0.001 ) and ( $p$ value $\leq 0.001$ ). Sensitivity and specificity of mammogram and ultrasound in differentiation between benign and malignant causes of pathologic nipple discharge were $71.4 \%$ and $54.2 \%$ respectively with positive predictive value of $31.2 \%$, negative predictive value of $86.7 \%$, and accuracy of $58.1 \%$. MRI gave higher sensitivity and specificity of $100 \%$ and $83.3 \%$ with positive predictive value of $63.6 \%$, negative predictive value of $100 \%$, and accuracy of $87.1 \%$.
\end{abstract}

Conclusion: Magnetic resonance imaging is superior to sonommagraphy in diagnosis of pathologic nipple discharge and we recommend it in special situations.

Keywords: Nipple discharge, Breast, MRI, Sonomammography

\section{Background}

Pathologic nipple discharge is characterized by spontaneous secretions from unilateral single or several ducts. A clear or bloody discharge is of major significance due to its higher association with breast masses [1]. Benign conditions such as papilloma or ductal ectasia are the commonest causes of most unilateral bloody nipple discharge. A rare but major cause of spontaneous pathologic nipple discharge (SPND) is breast cancer which accounts for $7-33 \%$ of the cases [2].

Traditional imaging methods used in the diagnostic approach to SPND are mammography and ultrasonography (US). Mammography is of limited use in cases of bloody nipple discharge, as it is often negative and fails to suggest the causative lesion. Not always microcalcifications associate the underlying process and sometimes masses are so small, so the sensitivity of mammography in these cases is inadequate [3].

Ultrasound of the breasts should be conducted in addition to mammography, as this modality allows for the detection of intraductal papilloma, particularly with the use of high-frequency probes (11-13 MHz) [4].

Magnetic resonance imaging (MRI) is a valuable adjunctive means of detecting and diagnosing papillomas and malignancies especially invasive ductal carcinoma and ductal carcinoma in situ (DCIS), especially in cases when the other two modalities are normal. In recent years, MRI has increased in importance in high-risk patients with nipple discharge and in patients with suspicious nipple discharge [5].

Our aim in this study was to determine the added value of dynamic contrast-enhanced MRI in diagnosis of different causes of pathologic nipple discharge.

\footnotetext{
* Correspondence: mokadem83@yahoo.com

'Department of Radiology, Mansoura University, Elgomhoria St., Dakahlia

35516, Egypt

Full list of author information is available at the end of the article
} 


\section{Methods}

\section{Patients (subjects)}

This is an institutional review board approved prospective study carried out in the period between January 2015 and August 2018. The study was conducted on 93 females complaining of spontaneous nipple discharge, their ages ranging from 27 to 70 years old with mean age of $49.74 \pm 11.81$. All patients gave an informed consent before inclusion in the study. Patients were subjected to full clinical examination, sonomammographic evaluation, post contrast dynamic MRI, and pathological evaluation. The inclusion criteria include non-lactating female complaining from spontaneous nipple discharge. The exclusion criteria were induced nipple discharge, any patient with contraindication for doing MRI, and pregnant female not candidate for mammography or post contrast MRI. Patients' demographics, nature, and side of the pathologic nipple discharge are described on Table 1.

\section{Imaging protocol}

Mammographic examinations were performed for only 84 patients (as the remaining nine patients were younger than 30 years old) using a LABDA/GMI (General Medical Italy) mammography system (LABDA/GMI, Italy). Standard craniocaudal (CC) and mediolateral oblique (ML) views were routinely obtained, and additional mammographic views were used as needed. The mammograms were assessed for the presence of mass (localized abnormal density), focal asymmetry, and calcifications.

Table 1 Demographic and clinical data of the studied group

\begin{tabular}{ll}
\hline Variables & $\begin{array}{l}\text { Study group }(n=93) \\
\text { No }(\%)\end{array}$ \\
\hline Age/years & $24(25.8)$ \\
$<40$ years & $30(32.3)$ \\
$40-50$ years & $39(41.9)$ \\
$>50$ years & $49.74 \pm 11.81$ \\
Mean \pm SD & $27-70$ \\
Range & \\
Color of discharge & $45(48.4)$ \\
Serous & $15(16.1)$ \\
Blood stained & $33(35.5)$ \\
Bloody & \\
Side & $39(41.9)$ \\
Right side & $42(45.2)$ \\
Left side & $12(12.9)$ \\
Bilateral & \\
Discharge & $51(54.8)$ \\
Induced & $42(45.2)$ \\
Spontaneous &
\end{tabular}

Breast US was performed by 10-year experienced radiologist (MMZ) using a scanner with 5-12 MHz lineararray transducers. US of both breasts was performed first by scanning each breast in the transverse and sagittal orientations, inner aspect of the breast in a supine position, and outer aspect in supine oblique position with the patient's arm raised above the head. The radiologist assessed the presence of breast masses, the lactiferous ducts and periductal area, and the axillary tail followed by scanning the axilla. In cases of palpable abnormality, targeted scanning at the area of concern was performed. US were assessed for presence of duct ectasia whether it is a simple duct ectasia or having echogenic content inside and intraductal breast masses. Masses were assessed for their number, size, shape, margin, echogenicity, and their distance from the nipple. Doppler scanning of the detected lesions was done to assess their internal vascularity.

MRI examinations were performed on a 1.5 - $\mathrm{T}$ system with a dedicated four-channel phased-array bilateral breast coil (Achieva, Philips Medical Systems, Best, the Netherlands). All patients were examined in the prone position with their arms elevated above the head. MR protocols include T1WI (TR $512 \mathrm{~ms}$, TE $8 \mathrm{~ms}$, slice thickness $3 \mathrm{~mm}$, FOV 400-500 mm, and matrix was $256 \times 256$ ), T2WI (TR $2000 \mathrm{~ms}$, TE $8 \mathrm{~ms}$, slice thickness $80 \mathrm{~mm}$, FOV 400-500 mm, and matrix was $256 \times 256$, flip angel $90^{\circ}$ ), and STIR (TR $4000 \mathrm{~ms}$, TE $70 \mathrm{~ms}$ and TI was $175 \mathrm{~ms}$, slice thickness was $3 \mathrm{~mm}$ with inter slice gap $1 \mathrm{~mm}$, FOV400-500 mm, and the matrix was $256 \times$ 256 were obtained). Dynamic images were taken after the administration of the contrast agent at $0.2 \mathrm{mmol} / \mathrm{L}$ per kilogram of body weight. Post contrast series, 1.16 min was the time between each study with 20-s break between the pre contrast and post contrast. Then a ROI was placed carefully within the most enhancing part of the lesion. The size of the ROIs were chosen in a lesion varied with the size and the shape of the lesion. MRI examination were analyzed by more than 15 years experienced radiologist (NYS) blinded from the sonomammographic findings for abnormal STIR signal intensity; presence of ductal, mass, or non-mass enhancement; shape; and size of detected lesions.

\section{Statistical analysis of the data}

We correlated the sonomammographic and MRI findings with pathological findings and compared sensitivity and specificity between sonomammography and MRI in detection of the underlying breast lesion.

Data were analyzed with SPSS ${ }^{\odot}$ V. 21 (IBM Corp., New York, NY; formerly SPSS Inc., Chicago, IL). The normality of data was first tested with Shapiro test. Qualitative data were described using number and percent. Association between categorical variables was tested using Chi-square test while Fischer exact test was used when expected cell 
count was less than 5. Continuous variables were presented as mean \pm SD (standard deviation) for parametric data and median (range) for non-parametric data. The two groups were compared with Student $t$ test for parametric data and Mann-Whitney test for non-parametric.

\section{Results}

The study included 93 female patients. Their age ranged from 27 to 70 years; mean age 49.7 years. All presented with nipple discharge; serous discharge (45 patients), serosanginous (15 patients), and bloody discharge (33 patients). Ultrasound and post contrast dynamic MRI were done to all patients, while mammography was done to 84 patients as the remaining nine patients were younger than 30 years old. Tissue pathology was done to all patients. Histopathological examinations revealed 69 benign lesions (74.2\%), three high-risk lesions (3.2\%), and 21 malignant lesions (22.6\%). Benign lesions were 36 duct ectasia (38.6\%), 24 duct papilloma (Fig. 1)/papillomatosis (25.8\%), six fibrocystic change (6.4\%), and three nipple adenoma (3.2\%); high risk lesions were three atypical epithelial hyperplasia (6.4\%) while malignant lesions were nine DCIS (9.7\%) (Figs. 2 and 3) and 12 intraductal carcinoma IDC (12.9\%) (Fig. 4). The histopathological types are summarized in Table 2.

Ultrasound findings include simple dilated ducts, ductectasia with echogenic content, postoperative changes, mottled parenchyma, and mass lesion. All patients presented by dilated ducts and echogenic contents inside were categorized as BIRADS 4a. Among these patients, 24 cases were papilloma/papillomatosis, three cases were atypical epithelial hyperplasia, while the remaining six cases were duct ectasia with inspissated secretions. On Doppler study, vascularity was seen in six cases (three papilloma and three atypical epithelial hyperplasia). Mammography was done in 30 out of 33 patients presented by ductectasia with echogenic components and different findings were detected including; dilated retroareolar duct (six patients), asymmetric
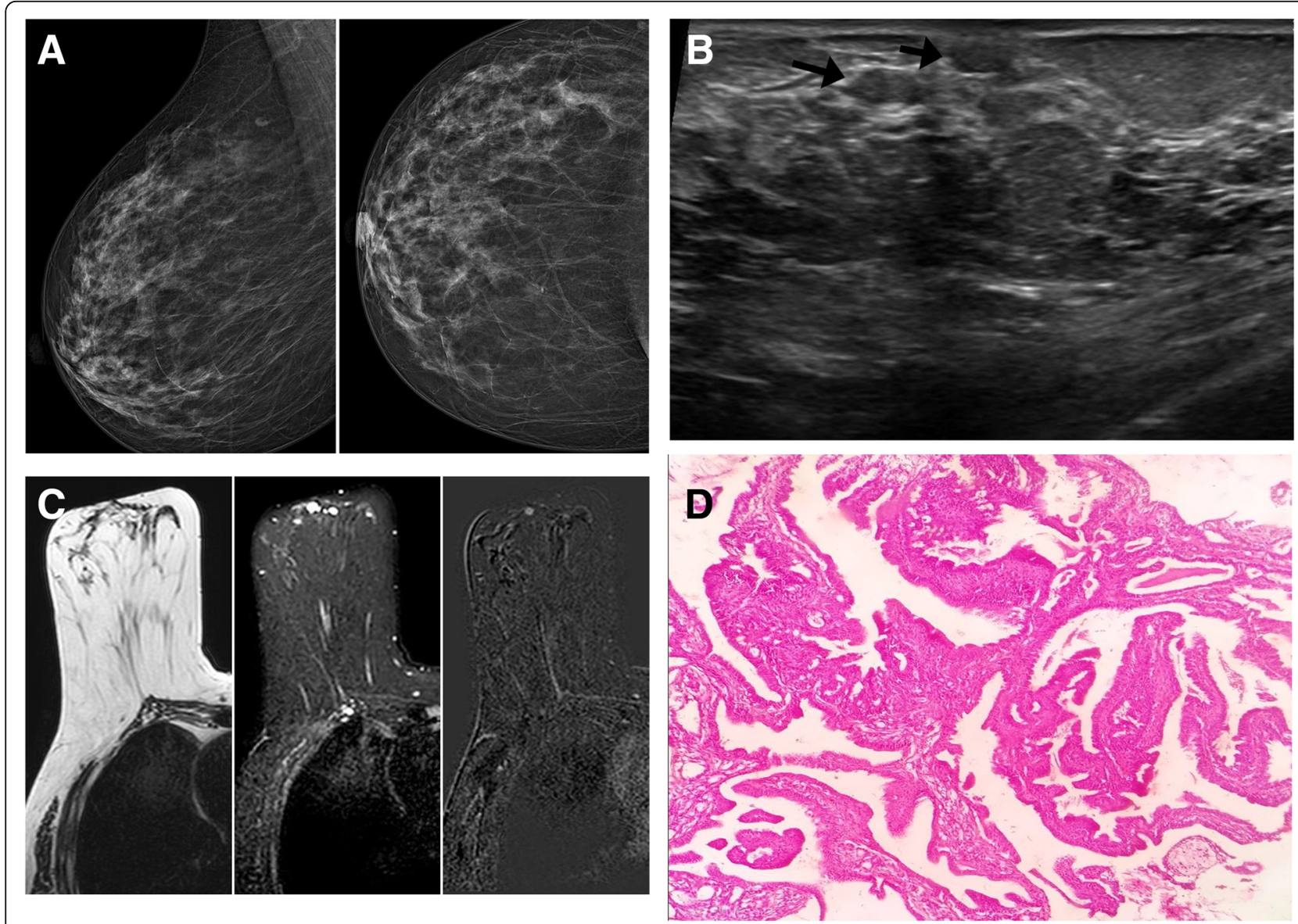

Fig. 1 A 49-year-old female patient with strong positive family history of breast cancer. a Mammography in mediolateral and craniocaudal views, showing no abnormality. b B-mode ultrasound image shows a small intraductal soft tissue in the right retroareolar region (black arrow). c T2, STIR, and contrast-enhanced T1 images show few small well circumscribed hyperintense lesions in the right retroareolar region. After IV contrast administration it shows enhancing focus in the right retroareolar region. $\mathbf{d}$ Histopathological results after excisional biopsy revealed multiple papillary formations showing complex arborizing pattern with well-developed connective tissue core surrounded by epithelial and myoepithelial cells. The lining cells have bland looking longitudinal nuclei with apocrine metaplasia features consistent with intraductal papilloma 

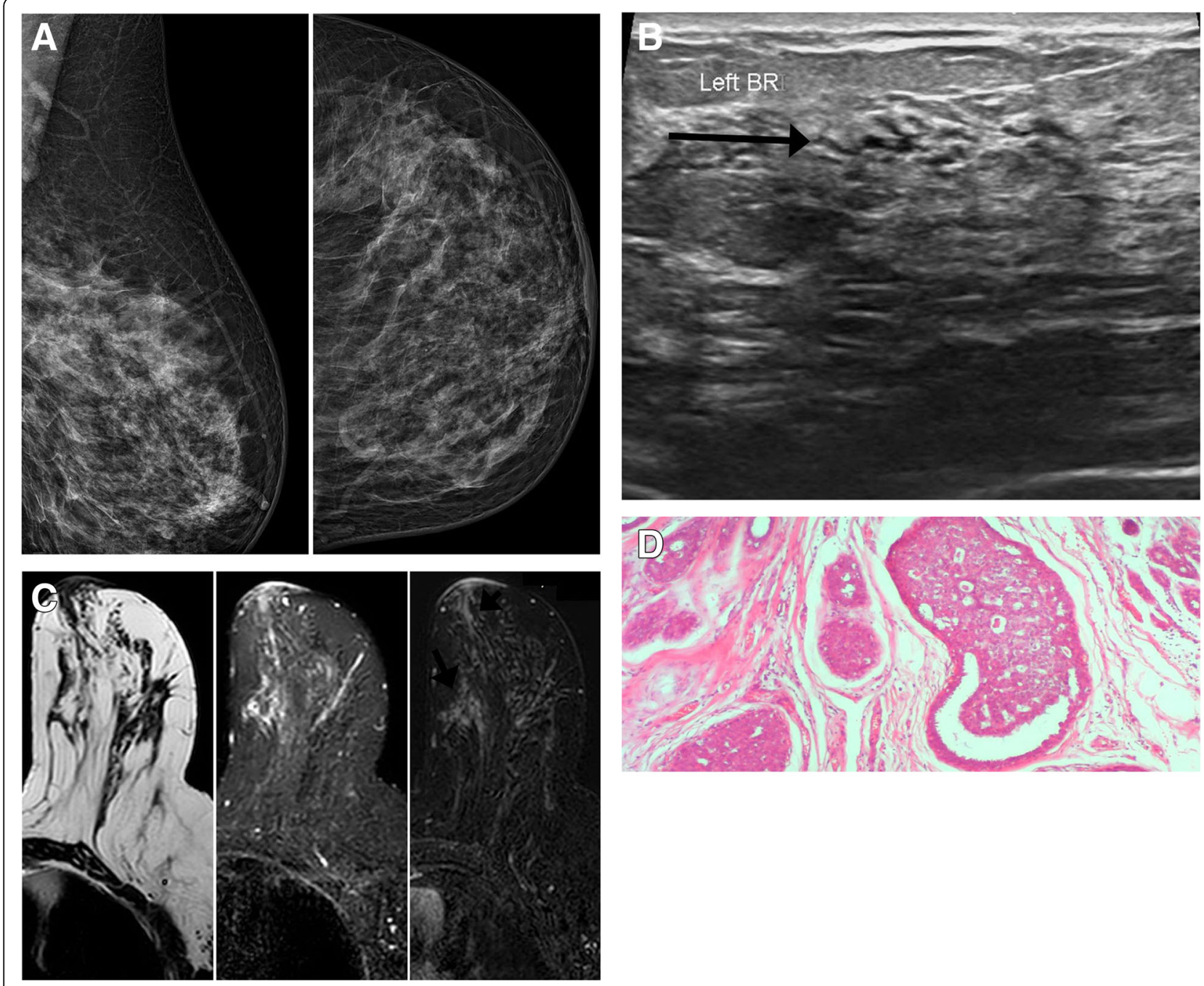

Fig. 2 A 59-year-old female patient complaining from palpable lump in the left upper inner quadrant. a Mammography in mediolateral and craniocaudal views, show moderate dense breast tissue. b B-mode ultrasound shows focal parenchymal heterogeneity (black arroe) corresponding to the clinically palpable lump. c T2, STIR, and contrast-enhanced T1 images; T2 image shows moderate glandular breast parenchyma while STIR image shows high SI area seen in the left upper inner quadrant, after IV contrast administration it shows non-mass clumped enhancement pattern (long black arrow) in the upper inner quadrant, also enhanced linear structure in the left retroareolar region (arrow head) extending to the nipple areolar complex. $\mathbf{d}$ Histopathology revealed ducts that are lined by monotonous round cell with mild increase in N/C ratio, small monotonous round nuclei with smooth contours, diffuse fine chromatin, indistinct nucleoli with rare mitotic figures consistent with low grade ductal carcinoma in situ

density (three patients), and no abnormality could be seen in the remaining 21 patients. All cases with positive findings were categorized as BIRADS 4a. On dynamic post contrast MRI, all of 33 cases were categorized as BIRADS 3. Enhancement was seen only in 27 out of these 33 cases (pathologically proved papilloma/papillomatosis and atypical epithelial hyperplasia) including mass enhancement (12 patients), focus enhancement (six patients), or non-mass enhancement (nine patients).

Nine cases of DCIS were pathologically proved in this study. On both mammography and complementary ultrasound, three cases were categorized as BIRADS 3 and the remaining six cases were categorized as BIRADS 4b. On dynamic contrast-enhanced MRI, all cases of carcinoma in situ were categorized as BIRADS 4 and appeared as non-mass enhancement.

The remaining 12 invasive carcinomas, nine cases of them were detected by both mammogram and US as suspicious masses (BIRADS $4 \mathrm{~b}$ and BIRDAS 4c), associated in situ component was occult in all cases by both US and mammogram, while the remaining three cases showed just postoperative changes and edema and were categorized as BIRDS 3. All cases and associated carcinoma in situ were detected by MRI and categorized as BIRADS 4 . The different imaging features in sonography, mammography, and MRI are described in Tables 3, 4, and 5 . 

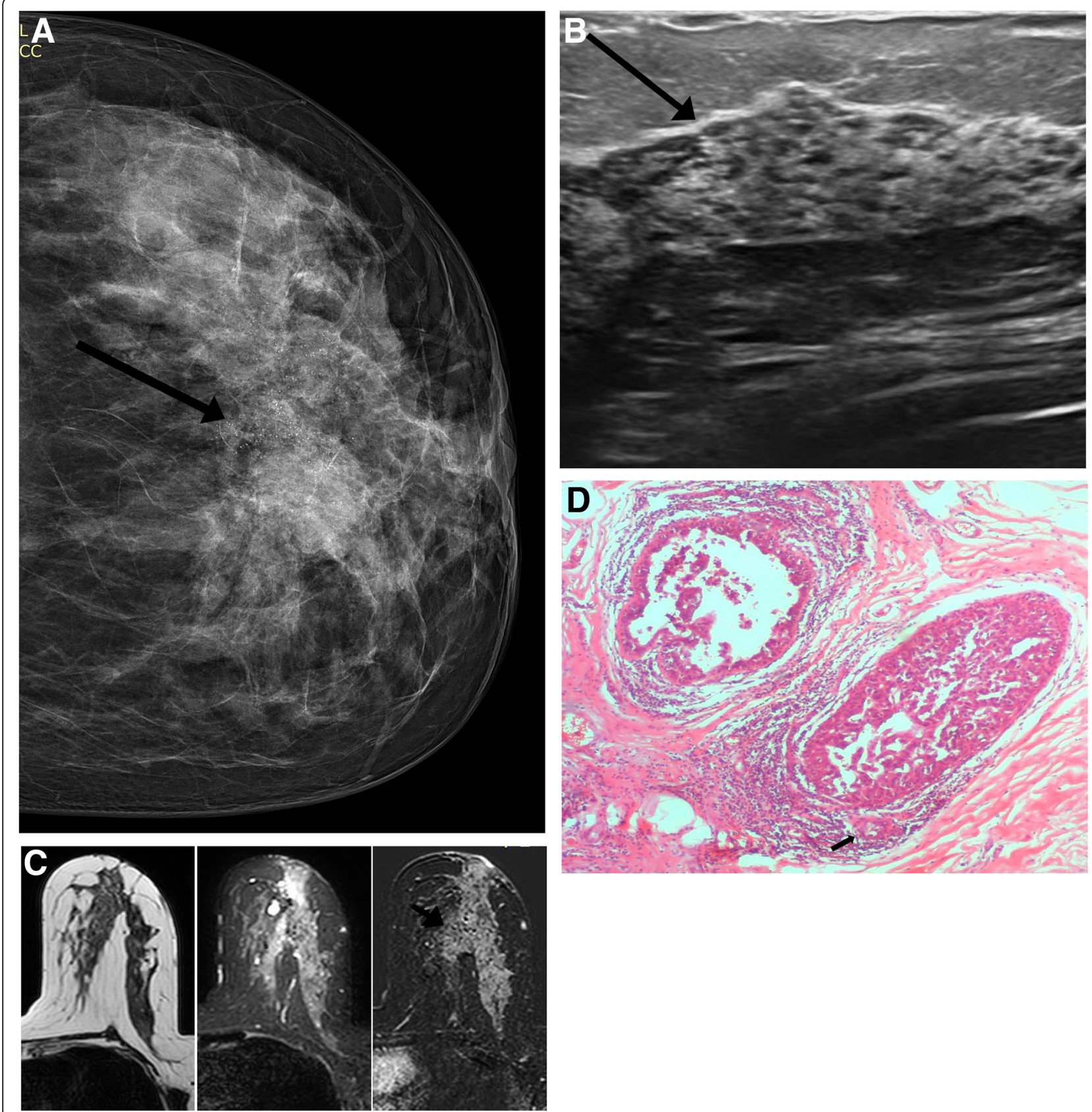

Fig. 3 A 52-year-old female patient with bleeding from left nipple. a Mammography in craniocaudal view shows focal asymmetry in the left retroareolar region with grouped microcalcifications (black arrow) that show segmental distribution. b B-mode ultrasound shows mottled parenchyma in the left upper outer quadrant (black arrow). c T2, STIR, and contrast-enhanced T1 images; T2 image shows moderate glandular breast parenchyma while STIR image shows low SI area in the upper outer quadrant extending from the retroareolar region to the posterior third of the breast. It shows non-mass enhancement after IV contrast administration with segmental distribution (black arrow). d Histopathology revealed high-grade ductal carcinoma in situ with focus of microinvasion in the form of nodule of invading neoplastic cells (not exceeding $1.0 \mathrm{~mm}$ ) into the periductal stroma, associated with fibroblast proliferation, collagenisation and focal inflammation

Simply dilated ducts and presence of a mass on US examination were of statistically significant probability in differentiation between benign and malignant causes of pathological nipple discharge ( $p$ value $=0.017$ and 0.001). Non-mass enhancement and STIR signal changes on MRI were of high statistically significant probability in differentiation between benign and malignant causes of pathological nipple discharge ( $p$ value $\leq 0.001$ ).

Sensitivity and specificity of mammogram and ultrasound in differentiation between benign and malignant 

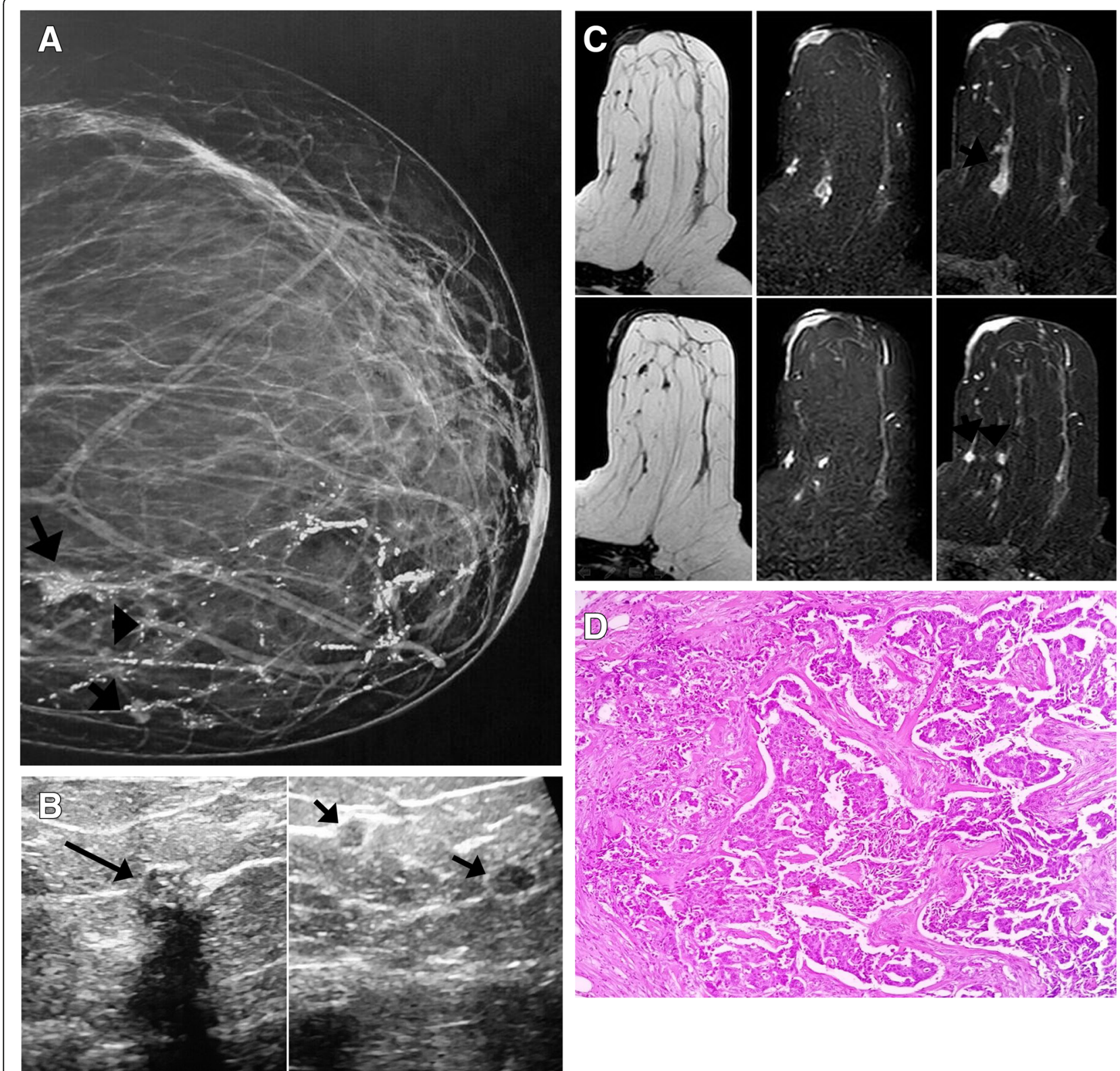

Fig. 4 A 40-year-old female patient complaining from bleeding from left nipple. a Mammography in craniocaudal view shows a partially circumscribed margin hyper dense mass seen in the left inner quadrant with another nearby two smaller similar mass lesions (marked by arrows). b B-mode ultrasound images show an irregular shaped mass seen in the left upper inner quadrant with another nearby smaller similar two masses (arrowed). c T2, STIR, and contrast-enhanced T1 images at different levels: T2 images show multiple low SI masses in the left upper inner quadrant that appear hyperintense on STIR images, after IV contrast administration, the masses display intense enhancement with enhancing left nipple (arrowed). d Histopathology shows sheets with attempt of tubular formations composed of atypical epithelial cells that display moderate degree of atypia, pleomorphism and mitotic activity. The neoplastic sheets are surrounded by desmoplasia consistent with grade II infiltrating ductal carcinoma

causes of pathologic nipple discharge were $71.4 \%$ and $54.2 \%$ respectively with positive predictive value of $31.2 \%$,negative predictive value of $86.7 \%$, and accuracy of $58.1 \%$,while sensitivity and specificity of $100 \%$ and $83.3 \%$ with positive predictive value of $63.6 \%$, negative predictive value of $100 \%$, and accuracy of $87.1 \%$. Table 6 shows validity of MRI and sonomammography compared to pathology.

\section{Discussion}

Nipple discharge accounts for $2-10 \%$ of the symptoms that women complain when they seek care at breast clinics and it is an alarming sign for both patients and clinicians since it may present sign of breast cancer [6]. In the evaluation of PND, the limitations of sonomammography and galactography have led to further research on complementary methods such as MRI and 
Table 2 Shows the different types of lesions

\begin{tabular}{lll}
\hline & Histological type & No (\%) \\
\hline Benign lesions $(n=69)$ & Duct ectasia & $36(38.6 \%)$ \\
& Duct papilloma/papillomatosis & $24(25.8 \%)$ \\
& Fibrocystic change & $6(6.4 \%)$ \\
& Nipple adenoma & $3(3.3 \%)$ \\
High risk lesions $(n=3)$ & Atypical epithelial hyperplasia & $3(3.3 \%)$ \\
Malignant lesions $(n=21)$ & DCIS & $9(9.7 \%)$ \\
& IDC & $12(12.9 \%)$ \\
\hline
\end{tabular}

ductoscopy [1]. So, in the workup and management of nipple discharge, conclusions drawn about the diagnostic utility of MRI [7].

Our study found that benign diseases $(74.2 \%)$ were the most common cause of pathologic nipple discharge, while we found high risk lesion and malignancy rate in (25.8\%) of cases. Paula et al. [8] reported that the most common causes of nipple discharge were some benign breast lesions, such as ductal ectasia, in $6-59 \%$ of cases, and papilloma, in 35-56\%. The risk of underlying malignancy is not negligible, ranging from 5 to $23 \%$. In the current study, most (72.3\%) of bloody nipple discharge was due to underlying malignant causes. Chen et al. [9] stated that there is association between the color of nipple discharge and breast cancer risk.

In our study, the mammographic findings in patients were as follows: asymmetric densities, dilated duct in sub areolar region, masses, and micro calcifications. Ballesio et al. [2] reported that mammography in patients with nipple discharge was negative in $60 \%$ of cases and also reported that the positive mammographic findings include asymmetric density, duct ectasia, and calcification.

The most common ultrasonographic features in this study were simple dilated ducts or duct ectasia with intraductal internal echoes. Ballesio et al. [2] found that the most common sonographic features in pathologic nipple discharge were duct dilatation, particularly associated with internal echoes and duct wall thickening. In our study, papilloma and papillomatosis on ultrasonography appeared as retroareolar dilated duct with

Table 3 Shows relation between US finding and pathology

\begin{tabular}{llll}
\hline US finding & $\begin{array}{l}\text { Malignant } \\
(n=21)\end{array}$ & $\begin{array}{l}\text { Benign } \\
(n=72)\end{array}$ & $p$ value \\
& No (\%) & No (\%) & \\
\hline Simple dilated ducts & $0(0)$ & $36(50)$ & $0.017^{*}$ \\
Duct ectasia with echogenic content & $3(14.3)$ & $33(45.8)$ & 0.201 \\
Postoperative changes & $3(14.3)$ & $1(1.4)$ & 0.226 \\
Mottled parenchyma & $3(14.3)$ & $1(1.4)$ & 0.226 \\
Mass lesion & $12(57.1)$ & $1(1.4)$ & $0.001^{*}$ \\
\hline
\end{tabular}

Table 4 Shows the different mammographic findings in 84 patients in correlation with the pathological findings

\begin{tabular}{|c|c|c|}
\hline Mammographic findings & No (\%) & $\begin{array}{l}\text { Pathological findings/ } \\
\text { results }\end{array}$ \\
\hline Negative & $45(53.6 \%)$ & $\begin{array}{l}\text {-Benign (42 cases) } \\
\text {-Malignant( } 3 \text { case) }\end{array}$ \\
\hline Masses & $15(17.9 \%)$ & $\begin{array}{l}\text {-Benign ( } 6 \text { cases) } \\
\text {-Malignant( } 9 \text { cases })\end{array}$ \\
\hline Dilated duct in subareolar region & $9(10.7 \%)$ & -Benign (9 cases) \\
\hline Asymmetric density & $9(10.7 \%)$ & $\begin{array}{l}\text {-Benign ( } 3 \text { case) } \\
\text {-Malignant( } 6 \text { cases })\end{array}$ \\
\hline Microcalcification & $6(7.1 \%)$ & $\begin{array}{l}\text {-Benign(3case) } \\
\text {-Malignant(3 case) }\end{array}$ \\
\hline
\end{tabular}

intraductal solid internal echoes. Mammography showed no abnormality, asymmetric density, retroareolar dilated ducts, and calcification. Eiada et al. [10] reported that small papilloma can be occult in mammography, particularly when located in the retroareolar regions because of the breast density and relative lack of compression in that area. Dhull et al. [11] reported that intraductal papillomas were seen as intraductal mass lesions in all patients on sonography and MRI, while were seen in only three patients (43\%) on mammography.

Lam et al. [12] found on US examinations dilated ducts associated with (21.7\%) of their detectable papillomata. A dilated duct is a sonographic sign useful for differentiating intraductal lesions from other benign lesions such as fibroadenoma. Papilloma may present as a circumscribed hypoechoic oval nodule. In this study, papilloma on MRI appeared as enhanced focus, enhanced mass, and non-mass enhancement with different kinetics making differentiation between papilloma and carcinoma is somewhat difficult. However, previous studies reported some overlaps in MRI findings between papilloma, papillomatosis, and carcinoma [10, 13].

As regard malignant causes of pathologic nipple discharge, mammographic findings of DCIS in our study were suspicious masses, focal asymmetry with or without calcifications. Yamada et al. [14] reported mass, asymmetries, and micro calcification in mammogram of DCIS. In our experience, ultrasonographic findings in DCIS were mottled/heterogeneous parenchyma with increased vascularity on Doppler, multiple intra cystic solid masses, and peripheral dilated ducts with irregular wall and echogenic contents. Watanabe $\mathrm{T}$ et al. [15] found that the most frequent ultrasonographic findings in DCIS were hypoechoic areas, followed by solid masses, abnormalities of the ducts or mixed masses, distortion, multiple small cysts, and echogenic foci without a hypoechoic area also were reported. The ultrasound features of DCIS have been described in the literature; however, the classifications vary slightly among authors. Ultrasound images of DCIS have been classified into 
Table 5 Shows relation between MRI finding and pathology

\begin{tabular}{|c|c|c|c|}
\hline & Malignant $(n=21)$ & Benign $(n=72)$ & $p$ value \\
\hline & No (\%) & No (\%) & \\
\hline Non-mass enhancement & $21(100)$ & $12(16.7)$ & $<0.001^{* *}$ \\
\hline Focal & $0(0)$ & $9(75)$ & \\
\hline Linear & $3(14.3)$ & $3(25)$ & \\
\hline Regional & $6(28.6)$ & $0(0)$ & \\
\hline Segmental & $9(42.8)$ & $0(0)$ & \\
\hline Diffuse & $3(14.3)$ & $0(0)$ & \\
\hline Enhanced focus & $0(0)$ & $3(4.2)$ & 1.0 \\
\hline Enhanced mass & $9(42.9)$ & $15(20.8)$ & 0.335 \\
\hline Shape of mass & & & 0.237 \\
\hline Rounded & $0(0)$ & $3(20)$ & \\
\hline Oval & $0(0)$ & $6(40)$ & \\
\hline Irregular & $9(100)$ & $6(40)$ & \\
\hline Margin of mass & & & 0.196 \\
\hline Circumscribed & $0(0)$ & $9(60)$ & \\
\hline Non circumscribed & $9(100)$ & $6(40)$ & \\
\hline No abnormal enhancement & $0(0)$ & $42(58.3)$ & $0.007^{*}$ \\
\hline STIR signal & & & $<0.001^{* *}$ \\
\hline No abnormal findings & $0(0)$ & $3(4.2)$ & \\
\hline Low signal mass & $9(42.9)$ & $6(8.3)$ & \\
\hline Low signal ducts & $0(0)$ & $18(25)$ & \\
\hline High signal mass & $0(0)$ & $12(16.7)$ & \\
\hline High signal ducts & $0(0)$ & $33(45.8)$ & \\
\hline High signal region & $12(57.1)$ & $0(0)$ & \\
\hline \multicolumn{4}{|l|}{ Size of lesion } \\
\hline Median width (range) & $6.5(1.5-9)$ & $3.7(0.75-6.7)$ & 0.275 \\
\hline Median Length (range) & $3(1.5-8.5)$ & $2.6(0.70-3.9)$ & 0.827 \\
\hline
\end{tabular}

masses, calcification alone, ductal change, and architectural distortion [16-20]. In our study, MRI findings of DCIS were retroareolar region of high signal intensity on T2WI, on dynamic post contrast study, diffuse non-mass enhancement with clustered ring pattern, segmental non-mass enhancement with clumped pattern, and regional non-mass enhancement. The extension of enhancement pattern was very close to the actual involvement of breast parenchyma by malignancy in correlation with pathology. So we found that MRI is valuable not only in diagnosis of DCIS but also for detection of actual extent of DCIS. Several reports [21, 22] found

Table 6 Shows validity of MRI and sonomammography compared to pathology

\begin{tabular}{llllll}
\hline & Sensitivity & Specificity & PPV & NPV & Accuracy \\
\hline MRI & $100 \%$ & $83.3 \%$ & $63.6 \%$ & $100 \%$ & $87.1 \%$ \\
Ultrasonography & $71.4 \%$ & $54.2 \%$ & $31.2 \%$ & $86.7 \%$ & $58.1 \%$ \\
\hline
\end{tabular}

that DCIS most commonly appears as a non-mass enhancement $(60-81 \%)$, and less frequently as a mass (14$41 \%)$ or a focus $(1-12 \%)$. Nori et al. [23] compared the mean absolute error between preoperative MRI and mammography evaluations and final disease extent; MRI showed an improved accuracy of $51.2 \%$. Study done by Hajaj et al [24] suggests that MRI could be an important tool in reducing the re-excision rates in the surgical management of high-grade DCIS.

In our experience, most of the infiltrating duct carcinoma cases were (IDC) associated with DCIS, and most cases were detected by mammogram and ultrasound, apart from few cases which were negative by mammogram due to dense parenchyma. Their appearance on US included irregular-shaped mass(es) with noncircumscribed margin and the remaining cases showed slight increase in echogenicity at the operative bed with nearby fluid collections and were categorized as BIRADS3 (postoperative change) as patients underwent 
unplanned resection for a mass. On MRI, all cases were detected, so MRI was valuable in detecting missed malignancy especially in dense parenchyma.

Bahl et al. [7] demonstrated that breast MRI is a valuable additional diagnostic tool in the setting of negative conventional imaging. In addition, a negative MRI in this symptomatic population may obviate the need for invasive surgery. Meta-analysis study done by Houssami et al. [25] reviewing 19 studies of women with breast cancer found that MRI detects additional disease in 16\% of women. Regarding MRI feature of IDC with DCIS, all masses were irregular in shape with non-circumscribed margin and they showed either heterogeneous or rim enhancement with associated non-mass enhancement of clumped pattern on post contrast study. Apple et al. [26] concluded that invasive ductal carcinoma on post contrast study appeared as enhanced irregular shaped mass with non-circumscribed margin, or may appear as nonmass enhancement.

In our study, sensitivity and specificity of mammogram and ultrasound in diagnosing cases with pathologic nipple discharger was $71.4 \%$ and $54.2 \%$ respectively, while sensitivity and specificity of MRI was $100 \%$ and $83.3 \%$. In a study done by Yllmaz $\mathrm{R}$ et al. [1], the sensitivity of US was found higher in the identification of intraductal lesions than that in the literature [20,21]. This study revealed sensitivity and specificity for US as $75 \%$ and $66.7 \%$, respectively. Ohlinger $\mathrm{R}$ et al. [27] reported a sensitivity of $82.9 \%$, higher than study done by Yilmaz R et al. [1], but they calculated a specificity of $17.9 \%$, which was lower than many studies. Ohlinger $\mathrm{R}$ et al. [27] study was multicentered, so it is difficult to standardize diagnostic criteria; this high rate of sensitivity could in part be due to broad positive findings (e.g., cystic lesions and ductal ectasia). Study done by Yilmaz R et al. [1] showed that US sensitivity and specificity can be higher if used specifically for ID pathologies and PND.

\section{Conclusion}

We can conclude that MRI add in diagnosis of different causes of pathologic nipple discharge and we believe it is mandatory in the following situations:

- If there is intraductal echogenicity to confirm intraductal mass lesion and exclude inspissated secretion especially in young female to preserve duct system and to differentiate between benign from suspicious lesion.

- In case of asymmetric heterogeneous breast parenchyma.

- In suspicious breast mass to determine the extent of the lesion for proper selection of patient for conservative management.
- Evaluation of residual suspicious lesion after improper operation.

\section{Abbreviations}

BI-RADS: Breast Imaging-Reporting and Data System; CC: Craniocaudal; DCE: Dynamic contrast enhanced; DCIS: Ductal carcinoma in situ; IDC: Intraductal carcinoma; MLO: Mediolateral oblique; SPND: Spontaneous pathologic nipple discharge

\section{Acknowledgments}

Not applicable.

\section{Authors' contributions}

All authors have read and approved the manuscript. Study concept and design was proposed by NYS. Patient recruitment, follow up and acquisition of data by AS and AH. Elgomhoria St. 35516NYS. Pathologic analysis; MMZ. Analysis and interpretation of data; drafting of the manuscript was done by AHE and MMz. Revision of the manuscript; AHE and NYS. Technical, or material support; AS, MMZ, and NYS.

\section{Funding}

This study did not receive funding.

\section{Availability of data and materials}

All data generated or analyzed during this study are included in this published article.

\section{Ethics approval and consent to participate}

Mansoura University institutional review board (IRB) approved this study. The proposal code is R.19.02.422. All procedures performed in studies involving human participants were in accordance with the ethical standards of the institutional and/or national research committee and with the 1964 Helsinki declaration and its later amendments or comparable ethical standards. A written informed consent was obtained from all individual participants included in the study."

\section{Consent for publication}

The participants in the study were informed and consented the possibility of research publication. Authors hereby transfer, assign, or otherwise convey all copyright ownership to the EJRNM in the event that such work is published in that journal.

\section{Competing interests}

The authors declare that they have no competing interest.

\section{Author details}

'Department of Radiology, Mansoura University, Elgomhoria St., Dakahlia 35516, Egypt. ${ }^{2}$ Department of Pathology, Mansoura University, Elgomhoria St., Dakahlia 35516, Egypt. ${ }^{3}$ Department of Surgery, Mansoura University, Elgomhoria St., Dakahlia 35516, Egypt.

Received: 14 September 2019 Accepted: 3 December 2019

Published online: 17 December 2019

\section{References}

1. Yllmaz R, Bender O, Yabul FC et al (2017) Diagnosis of nipple discharge: value of magnetic resonance imaging and ultrasonography in comparison with ductoscopy. Balkan Med J 34:119-126

2. Ballesio L, Maggi C, Savelli S et al (2008) Role of breast magnetic resonance imaging (MRI) in patients with unilateral nipple discharge: preliminary study. Radiol Med 113:249-264

3. Lorenzon M, Zuiani C, Linda A et al (2011) Magnetic resonance imaging in patients with nipple discharge: should we recommend it? Eur Radiol 21: 899-907

4. Zervoudis S, latrakis G, Economides P et al (2010) Nipple discharge screening. Womens Health (LondEngl) 6(1):135-151

5. Lubina N, Schedelbeck U, Roth A et al (2015) 3.0 Tesla breast magnetic resonance imaging in patients with nipple discharge when mammography and ultrasound fail. Eur Radiol 25:1285-1293

6. Patel BK, Falcon S, Drukteinis I (2015) Management of nipple discharge and the associated imaging findings. Am J Med 128:353-360 
7. Bahl M, Baker JA, Green up RA et al (2015) Evaluation of pathologic nipple discharge: what is the added diagnostic value of MRI? Ann Surg Oncol 22: 435-441

8. Paula IB, Campos AM (2017) Breast imaging in patients with nipple discharge. Radiol Bras 50(6):383-388

9. Chen L, Zhou WB, Zhao Y et al (2012) Bloody nipple discharge is a predictor of breast cancer risk: a meta-analysis. Breast Cancer Res Treat 132(1):9-14

10. Eiada R, Chong J, Kulkarni S et al (2012) Papillary lesions of the breast: MRI, ultrasound and mammographic appearances. AJR Am J Roentgenol 198: 264-271

11. Dhull V, Sen J, Yadavet R et al (2016) Case series: imaging features of intraductal papillomas in patients presenting as nipple discharge. Int J Res Med Sci 4(7):2878-2882

12. Lam WWM, Chu WCW, Tang APY et al (2006) Role of radiologic features in the management of papillary lesions of the breast. Am J Roentgenol 186(5): $1322-1327$

13. Sarica O, Ozturk E, Demirkurek HC et al (2013) Comparison of ductoscopy, galactography, and imaging modalities for the evaluation of intraductal lesions: a critical review. Breast Care 8(5):348-354

14. Yamada T, Mori N, Watanabe M et al (2010) Radiologic-pathologic correlation of ductal carcinoma in situ. RadioGraphics 30:1183-1198

15. Watanabe T, Yamaguchi T, Tsunoda $Y$ et al (2017) Ultrasound image classification of ductal carcinoma in situ (DCIS) of the breast: analysis of 705 DCIS lesions. Ultrasound Med Biol 43(5):918-925

16. Cho KR, Seo BK, Kim CH et al (2008) Non-calcified ductal carcinoma in situ: ultrasound and mammographic findings correlated with histological findings. Yonsei Med J 49:103-110

17. Mesurolle B, El-Khoury M, Khetani K et al (2009) Mammographically noncalcified ductal carcinoma in situ: sonographic features with pathological correlation in 35 patients. Clin Radiol 64:628-636

18. Mun HS, Shin HJ, Kim HH et al (2013) Screening-detected calcified and noncalcified ductal carcinoma in situ: differences in the imaging and histopathological features. Clin Radiol 68:27-35

19. Park JS, Park YM, Kim EK et al (2010) Sonographic findings of high-grade and non-high-grade ductal carcinoma in situ of the breast. J Ultrasound Med 29:1687-1697

20. Wang LC, Sullivan M, Du H et al (2013) Appearance of ductal carcinoma in situ. Radiographics 33:213-228

21. Ballard L, Ballard GR (2013) High-grade ductal carcinoma in situ: an overview for the radiologist. J Am Osteopath Coll Radiol 2:18-25

22. Greenwood HI, Heller SL, Kim S et al (2013) Ductal carcinoma in situ of the breasts: review of MR imaging features. Radiographics 33:1569-1588

23. Nori J, Meattini I, Giannotti E et al (2014) Role of preoperative breast MRI in ductal carcinoma in situ for prediction of the presence and assessment of the extent of occult invasive component. Breast J 20(3):243-248

24. Hajaj M, Karim A, Pascaline $S$ et al (2017) Impact of MRI on high grade ductal carcinoma Insitu (HG DCIS) management, are we using the full scope of MRI? EJR 95:271-277

25. Houssami N, Ciatto S, Macaskill P et al (2008) Accuracy and surgical impact of magnetic resonance imaging in breast cancer staging: systematic review and meta-analysis in detection of multifocal and multicentric cancer. J Clin Oncol 26(19):3248-3258

26. Apple S, Bassett L, Poon C et al (2011) Invasive ductal carcinomas. In: Breast Imaging, 1st edn. Elsevier, Philadelphia. p 423

27. Ohlinger R, Stomps A, Paepke S et al (2014) Ductoscopic detection of Intraductal lesions in cases of pathologic nipple discharge in comparison with standard diagnostics: the German multicenter study. Oncol Res Treat 37:628-632

\section{Publisher's Note}

Springer Nature remains neutral with regard to jurisdictional claims in published maps and institutional affiliations.

\section{Submit your manuscript to a SpringerOpen ${ }^{\circ}$ journal and benefit from:}

- Convenient online submission

- Rigorous peer review

- Open access: articles freely available online

- High visibility within the field

- Retaining the copyright to your article

Submit your next manuscript at $\boldsymbol{\nabla}$ springeropen.com 